\title{
$\widehat{A}$ Madridge \\ madridge Journal of Nursing \\ Interconnecting Scientific World
}

Research Article

Open Access

\section{Non-Suicidal Self-Injury Risk Screening in Primary Care Settings: Results of two Instrument Development Studies}

\author{
Kimberly A Williams ${ }^{1 \star}$, Lynette Phillips ${ }^{2}$, Jara Chadwell ${ }^{1}$, and Hannah Monsman ${ }^{1}$ \\ ${ }^{\prime}$ College of Nursing, Kent State University, Kent, OH, USA \\ ${ }^{2}$ College of Public Health, Kent State University, Kent, OH, USA
}

\section{Article Info \\ *Corresponding author: Kimberly A Williams \\ College of Nursing \\ Kent State University \\ P.O. Box 5190 Kent, OH 4424 \\ USA \\ E-mail: kwill190@kent.edu}

Received: December 12, 2017

Accepted: February 11, 2018

Published: February 19, 2018

Citation: Williams KA, Phillips LS, Chadwell J, Monsman H. Non-Suicidal Self-Injury Risk Screening in Primary Care Settings: Results of two Instrument Development Studies. Madridge J Nurs. 2018; 3(1): 92-100.

doi: 10.18689/mjn-1000117

Copyright: @ $₫ 2018$ The Author(s). This work is licensed under a Creative Commons Attribution 4.0 International License, which permits unrestricted use, distribution, and reproduction in any medium, provided the original work is properly cited.

Published by Madridge Publishers

\begin{abstract}
Negative experiences during childhood can lead to adverse outcomes such as behavioral problems and mental health disorders including non-suicidal self-injury. Screening in primary care settings can improve early assessment, intervention, and treatment. This article describes both a state-wide school nurses' study and a chart review study that use two instruments, a self-administered tool and a clinician administered tool, for screening of non-suicidal self-injury in primary care settings. Previous instrument development studies were conducted for validity and evaluation of the sensitivity of the clinician administered tool to screen against four risk categories (no risk, low risk, moderate risk and high risk). The school nurse study demonstrated testretest reliability for both tools. The chart review study demonstrated mixed findings. More studies conducted with larger clinical populations and comparison tools are certainly needed.
\end{abstract}

Keywords: Non-suicidal self-injury; Instrument development; Screening.

\section{Introduction}

Non-suicidal self-injury (NSSI) behaviors are a significant problem in the United States and worldwide [1-3] as they are commonly associated with depression, anxiety, and other mental health issues. Mental health disorders are more prevalent among families who experience early life stressors [4]; this relationship has been documented in children as young as three years of age, is cumulative, and continues throughout their school years $[1,5,4]$. Cumulative stressors can lead to poor coping mechanisms which can trigger NSSI behaviors [6,7]. The antecedents and consequences of NSSI behaviors dictate a need to develop easy to use screening tools to establish the presence and severity of NSSI and to identify risk factors for these behaviors. Thus, the purpose of this article is to describe two instrument development studies using two screening instruments that may be useful for identifying children and adolescents who are at risk for or exhibit existing NSSI behaviors.

\section{Background}

Non-suicidal self-injurious behaviors are intentional, direct, and deliberate selfdestruction of body tissue without suicidal intent [8]. The range of non-suicidal selfinjury behaviors includes cutting, carving, burning, scalding, picking, scratching, interfering with wound healing, head banging, pulling of any hair on the body, severe nail biting, skin biting, needle sticking, and inserting foreign objects into the skin or 
body cavities $[9,10]$. In addition to these types of self-injury behaviors, some of the literature has suggested that excessive tattoos and piercings are also forms of NSSI behaviors [11,12], while other researchers argued that motivations for these types of behaviors are forms of self-expression and are not related to negative thoughts and self-harm [13,14].

The prevalence rate for NSSI in adolescent clinical samples ranges from $14 \%$ to $46 \%$ [7,15-23]. Historically, studies indicated that NSSI behavior occurred more often in females, whites/Caucasians, and those with mood disorders and anxiety $[5,16]$ However, newer studies have presented inconsistent findings regarding gender and race/ethnicity, requiring further research [24]. Non-suicidal self-injurious behaviors generally begin during adolescence between the ages of 11 and $19[7,9$, $25-31]$ and gradually increase as grade level increases $[16,20]$. One rationale for the age of initiation and increase in NSSI is related to coping skills development during adolescent years. Adolescents with NSSI behaviors may have difficulty identifying and describing their emotions and have poor awareness of body cues [15]. The inability to express emotions and limited awareness of body cues during this significant developmental period places them at higher risk for development of NSSI behaviors [15]. Specifically, adolescents who reported NSSI behaviors expressed feelings of guilt and shame, teasing by peers, and parental distress [32].

Although there is no intention of suicide with NSSI behaviors, it remains a higher risk for this group. Attempted suicide in the general adolescent population reporting at least one attempt in the past year was approximately $6.3 \%$ [33]. However, rates of attempted suicide in clinical samples of adolescents reporting histories of both NSSI behaviors and attempted suicide were $14-70 \%$ [34], it has been theorized that higher rates may be attributed to reduction in fear of suicide through NSSI behaviors [35]. The potential squeal of NSSI supports the need for early identification and intervention.

According to the National Alliance on Mental IIIness [36], schools hold the largest population of the children in the community. Therefore, screenings may be most effective when implemented in the school setting. However, existing tools may be limited, research based, or included as a part of suicide assessments [37]. These tools have been extremely helpful in understanding the components that are inclusive to NSSI behavior. For example, to understand non-suicidal behavior a person may ask questions such as: How often do you harm yourself? How do you harm yourself? What does harming yourself feel like? What do you like about harming yourself? What do you not like about harming yourself? Why do you harm yourself? These questions are important for laying the foundation for understanding the behavior but may not address the need for early screening for case finding for treatment [38]. More recently, assessment tools have been developed to screen for NSSI behavior. The Non-Suicidal SelfInjury-Assessment Tool (NSSI-AT) and the brief version do screen for NSSI behaviors with two questions "have you ever done any of the following", then listing fifteen NSSI behavioral options from choose to choose. The second question asks for any other ways the person tried to physically harm or mutilate them self. Another instrument, the Self-Injurious Thoughts and Behavioral Inventory (SITBI) had one NSSI question. "Have you ever done something to purposely hurt yourself without intending to die?" Therefore, only a few tools assess NSSI behavior for the purpose of screening, case finding, and treatment referral existing.

\section{Pre-Clinical Development of Screening Tools}

\section{Development of the Initial Tool}

A literature review was conducted in 2007 to understand NSSI behavior. Because our initial search occurred two years prior to the publication of the term NSSI by Nock and Favazza [10], our literature search was conducted using Pub Med and the terms self-mutilation and adolescent with search dates between 1990 and 2007. There results of the search, published in 2007 [39] yielded 21 articles specifically related to NSSI [38, 39]. After the initial literature review, each article was reviewed for common risk factors and behaviors resulting in the identification of 39 factors related to NSSI which were pooled into categories. Researchers created an acronym for each category of the tool called SLICE: "S" = Scars/wounds, especially on the extremities; "L" = Links to commonly associated risk factors such as stress, depression, sexual abuse, and other social determinant items; "I" = Injuries that do not match the reported history associated with the injury; " $\mathrm{C}$ " = Clothing that appears to be non-seasonal/culturally inappropriate and used to conceal wounds; and " $E$ " = Environment that may be risky or abusive and other social determinant items [40]. This framework was used to develop the clinician administered assessment tool. To support content validity, four health providers reviewed the initial clinician-administered risk screening tool: a clinical nurse researcher, a PhD psychologist, a child and adolescent psychiatrist, and a family psychiatric nurse practitioner (NP).

In 2017, a similar literature review was conducted to assess for new research findings of NSSI behavior using the CINHAL, Pub Med, Medline, Psych INFO, Healthsource databases, and a Google scholar internet search. This literature review was conducted using the search terms young adult and non-suicidal self-injury for articles published between 2007 and 2017. Articles included were peer reviewed, conducted in the U. S., participants under 35 years, discussed factors of non-suicidal self-injury, and published in English. The initial search outcome generated 233 results. After excluding 71 duplicates, the titles and abstracts of 162 studies were read. Articles that focused on a different psychopathology, treatment, clinically induced pain, media perceptions, or acute vs. chronic NSSI were also excluded resulting in 43 studies. While most of the factors found in the 2007 literature search remained constant, studies that involved gender and racial/ ethnic differences were inconsistent in newer study findings [24]. 
With IRB approval, subsequent testing of the screening tool occurred over six studies. Four studies were conducted for refinement of the screening tools using non-clinical settings with a fifth study conducted to further validate and test the reliability of the screening tool using a large population. The sixth study was the first to use a clinical population. Both studies are described in more detail later in this article.

\section{Hypothetical Case Studies}

In the first study, researcher's created hypothetical cases derived from previously known and de-identified patient exemplars with medical and/or mental health disorders. The second study used both high- and low-risk case exemplars for further refinement. In the third study, the participants recommended the development of a self-administered assessment tool as a pre-screening for the clinician administered risk screening tool. For the fourth [40], a moderate risk hypothetical case was included to improve instrument sensitivity. Participants for these first four studies included registered nurses who were psychiatric or family nurse practitioner students and school nurses.

\section{Study 5 Materials and methods}

The purpose of the fifth study, a mixed method approach, was to further validate and test the reliability of a self-report questionnaire (See Appendix A) and clinician administered screening tool (See Appendix B) for identification of risk for NSSI using a statewide school nurse population. Approval for the study was given by the University of South Alabama institutional review board and the Alabama Department of Education in 2012. The research questions were: 1) will school nurses validate the items as important in the assessment of NSSI for both tools? And 2) will school nurses who complete both tools using hypothetical cases score similar at times 1 and 2 demonstrating reliability?

\section{Mixed Methods Qualitative Component}

For the qualitative component, a school nurse focus group was conducted by the researchers. One researcher's role was as a scribe, while the other led the focus group using the following questions:

1. Have you previously cared for a child/adolescent with self-injury or self-mutilation?

2. What is the strongest feature of the tools?

3. What is the weakest feature of the tools?

4. What modifications (if any) would you suggest to the content?

5. Is there any material that should be eliminated as not essential?

6. Please comment on the organization of the tools.

7. Please give your overall impression of the tools.

8. Do you have any further comments or recommendations?
A flyer was then sent to school nurses within a local county school system to recruit participants. Six school nurses responded by e-mail that they would participate and consent was obtained at the beginning of the focus group. Each participant received $\$ 30$ at the end of the focus group.

\section{Qualitative Results}

Of the six school nurses who participated in the focus group, all had experience with school age children with NSSI. There were five females and one male who participated. The racial and ethnic demographics were African American $(\mathrm{N}=3)$ and Caucasian $(\mathrm{N}=3)$. Ages ranged from 31 to 65 with a mean age of 43. After consent was obtained, participants were given both tools to review with the list of questions. Researchers discussed each question, recorded the group's responses, and then reviewed the comments. For the self-report questionnaire, the participants recommended inclusion of a check box to indicate if the children/adolescence had difficulty reading and needed help to complete the form. They also stated that they liked the wording with each question on the form of "how true is this for you? "When asked about the clinician administered screening tool scoring, the group commented that they understood the directions for scoring and described the clinician-administered screening tool as "user-friendly." They also recommended bold lines to separate the sections of this tool. They proposed revising the wording for the injuries section of the clinician administered screening tool for better clarification and to add an item asking if the student was under the care of a doctor or therapist. The group felt that IQ levels were important regarding students at risk for self-injury. Another high-risk factor seen by the school nurses was the unknown location or incarceration of a parent.

The group discussed the clinician screening tool section regarding information about clothing and risk. They shared that students at some schools wear mostly uniforms. Also, students frequently wear jackets due to the classroom climate being excessively cold or to cover clothing in poor condition. They stated that some students wear belts with skulls or other symbols and draw on their uniforms. This could possibly be attributed to an identity of a group membership or Goth identity when dressing styles are limited to uniforms. When asked about excessive tattoos and body piercings as risk factors, the group added that they also see students with "Gauges" which is a type of jewelry for stretching healed body piercings.

Recurring key words were documented by the researchers during the group meeting and included disrupting, bullying, cyber bullying, low self-esteem, and lack of parental/adult supervisions of children. When the items of the tools and keywords were reviewed with the focus group, they agreed that both represented risk factors for NSSI. The group discussion then focused on what the school nurses had seen related to NSSI in their school system. Cases discussed included NSSI behaviors seen in five children, four females and one male, who were between the ages of 4 to 17 years. The racial/ethnic compositions of the cases discussed were 
African American and Caucasian. Notable factors in these children included a mother who was in jail, a father whose location was unknown, attending an inner city elementary school, being very quiet and having withdrawn behavior, being bullied, and having a low IQ. Behaviors the school nurses had seen in the children included skin picking, fascination with knives, outburst and disruption, mimicking cutting behaviors with crayons/pens, and use of NSSI behavior for seeking attention.

\section{Mixed Methods}

\section{Quantitative Component}

For the quantitative component, state school nurses were invited by a flyer to participate in a study to help refine the two tools. Consent was considered given by the school nurses' completion and return of the two tools. Participants received three hypothetical cases and the self-report questionnaire and clinician-administered screening tool to complete. They were asked to place themselves in the role of each hypothetical case and then complete the self-report questionnaire for each case. Next, they received three different hypothetical cases and the clinician-administered screening tool. They were asked to read and complete the clinician administered screening tool as if they were the nurse assessing the adolescent for each case. Two to four weeks later, they repeated the same cases for both tools again. At the end of the study, each participant was given $\$ 60$ and six continuing education credits.

\section{Results}

For the quantitative phase, 1,300 state school nurses were invited to participate with $8 \%(n=109)$ whom participated at time one and $7.8 \%(n=102)$ remained in the study at time two. The Wilcoxon signed rank test, a non-parametric test for differences in observations and appropriate when distribution is not considered normal, was chosen for statistical analysis. The test-retest for results for low, moderate and high cases of the SARA and SLICE are shown in Table 1, indicating no significant differences between time one and time two.

Table 1. P-Values for the Self-Report Questionnaire and Clinician Administered Risk Screening Tool

\begin{tabular}{|l|l|l|l|}
\hline Self-report & High Risk Case & Moderate Risk & Low Risk Case \\
\hline Go SLICE & $\mathrm{p}=1.0000$ & $\mathrm{p}=0.4020$ & $\mathrm{p}=0.4316$ \\
\hline Clinician-admin & High Risk Case & Moderate Risk & Low Risk Case \\
\hline Action & $\mathrm{p}=1.0000$ & $\mathrm{p}=0.1215$ & $\mathrm{p}=0.7500$ \\
\hline
\end{tabular}

\section{Discussion}

The review of reoccurring themes and key words of the school nurse focus group continued to support the findings of concepts related to NSSI behavior found in the literature. This study further validated the items of the tools for measuring the risk for NSSI. Further revisions were made as a result of this focus group. These changes included adding a question to the self-report questionnaire to indicate if the child had difficulty reading. Changes for the clinician administered screening tool included bolding of the lines of each section, retaining the item of excessive tattoos and body piercing, and including an item of being a victim of bullying. The $p$-values (See Table 1) were much larger than the significance; we conclude that there was no difference between time 1 and 2 . Indicating that participants completed the screening tools similarly for each case at two different time points. Thus, the findings of the test-retest demonstrated reliability of both tools with the use of hypothetical cases.

\section{Study 6 Materials and Methods}

The previous studies utilized hypothetical cases and clinical testing of the screening tools was needed. This study used a retrospective chart review methodology to assess the reliability of the self-administered risk assessment screening tool and the clinician administered risk screening tool to identify severity of behavior risks including NSSI behaviors within a clinical population. Approval for the study was given by the University of South Alabama institutional review board in 2013 and a local community mental health facility was approached and agreed to participate in this study. Additional approval by the Kent State University institutional review board was given for continued data analysis in 2016.A meeting was held to review the purpose and steps of the study with the facility director and psychiatric providers. Participants who were psychiatrists were recruited via e-mail through a flyer sent out by the director of the facility. There was no compensation for participation. Of the six psychiatrists on staff at the child and adolescent facility, four agreed to participate. Providers were asked to choose fifteen patients with NSSI behaviors and fifteen patients without NSSI behaviors $(n=30)$ for chart review. They reviewed the demographics, diagnosis, and severity level of each chart, indicated this in the required area of the data collection form, and then completed both tools. Because the method used for this study was a retrospective chart review process of patients who had been discharged, under the care of and well known to participants, they were asked to complete the self-report assessment tool (See Appendix A) as if they were the patient. Then, participants completed the clinician- administered risk screening tool (See Appendix B) in their role as a healthcare provider. Training for completing the assessment tools was provided by conducting a one-time meeting with the providers introduce the two tools and written instructions for the study and completing the tool was sent to each participant once consent was received.

Once the self-administered risk assessment tool was completed by the participants, a positive finding indicated the need for further screening by the clinician administered risk assessment tool. We assessed agreement of the psychiatrist and the researcher for indicating the "needing further evaluation" on the self-administered risk assessment tool using McNemar's chi square test. We then tested agreement of the psychiatrist and the researcher using a one-way ANOVA with post-hoc testing of the scores and risk categories (no risk, low 
risk, moderate risk, and high risk) for the clinician-administered screening tool. To better understand if the number of risk factors under the "Link" section (commonly associated risk factors such as stress, depression, sexual abuse, and other social determinant items and under the "Environment" section differed by risk category, we compared the mean score using an independent samples t-test. To evaluate the sociodemographics of the population who were previously or currently engaging in NSSI behaviors, we compared the presence of scars or wounds with age range, race, and gender categories using Pearson's chi-square test of independence.

\section{Results and Discussion}

Only one provider completed the study reviewing 28 charts for analysis. The results of the McNemar's chi square test of the self-administered risk assessment tool found complete agreement between the psychiatrist and the researcher regarding whether or not the case should be referred to the clinician-administered risk assessment tool. All cases $(n=28)$ screened using the clinician-administered risk assessment tool were deemed "moderate" or "high" risk by both the researcher and the psychiatrist. Two-thirds of the psychiatrist's assessments agreed with the risk category identified by the researcher, but the result was not statistically significantly different (See Table 2).

Table 2. Agreement between Psychiatrist and Researcher on Risk Category

\begin{tabular}{|l|c|c|}
\hline & $\mathrm{N}(\%)$ & $\mathrm{p}$-value \\
\hline Agreed on risk category & $19(67.9)$ & 0.45 \\
\hline Disagreed on risk category & $7(25.0)$ & \\
\hline Missing & $2(7.1)$ & \\
\hline
\end{tabular}

McNemar's Chi Square Test for Paired Data

We combined the psychiatrist's and the researcher's number of risk factors under the "Links" section and "Environment" sections to examine the mean scores for the risk categories. The high-risk category contained more risk factors found under the "Links" section compared to the moderate risk category(See Table 3) and the difference was statistically significant $(p<0.0001)$. There also was a statistically significant difference in mean number of risk factors under the "Environmental" section by risk category; however, the moderate risk group had a higher mean amount than the high risk group (See Table 4). We wanted to analyze the patient socio-demographic characteristics of patients for whom the chart review was completed. Examination of sociodemographic variables showed both gender and race were associated with number of scars or wounds, while age was not. Specifically, a higher proportion of females than males had two or more scars or wounds than males, and white/Caucasians had a much higher proportion of two or more scars or wounds than all other race/ethnicities (See Table 5).

Table 3. Mean Number of Risk Factors under Links Section by Risk Category

\begin{tabular}{|l|c|c|c|}
\hline Risk Category & $\mathrm{N}$ & Mean (SD) & p-value \\
\hline Moderate risk & 11 & $6.55(1.97)$ & $<0.0001^{\star}$ \\
\hline High risk & 47 & $10.1(2.06)$ & \\
\hline
\end{tabular}

*Statistical Significance using t-test
Table 4. Mean Number of Risk Factors under Environment Section by Risk Category

\begin{tabular}{|l|c|c|c|}
\hline Risk Category & $\mathrm{N}$ & Mean (SD) & p-value \\
\hline Moderate risk & 11 & $5.27(5.35)$ & $<0.0001^{\star}$ \\
\hline High risk & 47 & $3.09(2.20)$ & \\
\hline
\end{tabular}

*Statistical Significance using t-test

Table 5. Relationship between Presence of Scars or Wounds and Age, Race, and Gender Categories

\begin{tabular}{|l|c|c|c|c|}
\hline & \multicolumn{4}{|c|}{ Number of Scars or Wounds } \\
\hline & $\begin{array}{c}0 \\
(n=8)\end{array}$ & $\begin{array}{c}1 \\
(n=6)\end{array}$ & $\begin{array}{c}2+ \\
(n=15)\end{array}$ & $p$-value \\
\hline $\begin{array}{l}\text { Gender } \\
\text { Female }\end{array}$ & 7 & 1 & 12 & \\
Male & 1 & 5 & 3 & $0.007^{\star}$ \\
\hline Age & 0 & 2 & 1 & \\
10-12 years & 5 & 1 & 9 & 0.184 \\
$13-15$ years & 3 & 3 & 5 & \\
$16-19$ years & 3 & 2 & 14 & \\
\hline Race/Ethnicity & & $0.016^{\star}$ \\
White/Caucasian & 5 & 4 & 1 & \\
All others & 3 & 4 & & \\
\hline
\end{tabular}

*Statistical Significance Using Chi-square Test of Independence

\section{Conclusions}

As stated previously, the purpose of this article is to describe the development of two screening instruments that may be useful for identifying adolescents who have potential for or are exhibiting existing NSSI behaviors. In the fifth study, the two tools demonstrated validity and stability with the test-retest for reliability. However, there were a few limitations. Although six participants are an appropriate number for a focus group, only one focus group was convened for the statewide school nurses study. More focus groups may have resulted in more suggested changes for the tools. Additionally, only hypothetical cases of adolescent patients have been used and the tools were not tested on children or adolescents in a clinical population.

In the sixth study, characteristics of the population in relation to the mean number of risk factors under the "Links" section of the clinician-administered assessment tool did show a relationship between higher number of scars/wounds and gender and race suggesting that other characteristics, as well as being female and white may indicate a higher risk for nonsuicidal self-injury and is consistent with many finding in the literature. One limitation of the sixth study was that it was conducted in a community mental health facility; conducting this study within the school setting reviewing student charts may have yielded different results. Another limitation of this study included no significant difference in agreement between the psychiatrist's assessments of risk categories and those of the researcher. We concluded that this was most likely due to low power to detect a difference $(\mathrm{N}=30)$. Also, the chart-review was conducted in a high-risk population, which may have had an impact on the findings. Conducting a chart review within a primary care clinical setting could have allowed for more comparison of low risk and moderate risk populations. Training for they psychiatrists, consisting of a one-time meeting followed by emailing written instructions with the consent forms and screening tools. This type of training without a platform for asking additional questions may not have been 
adequate prior to the chart review. Also, we were not able to determine significance for the age comparison due to the small sample size and age of the population sampled (all charts reviewed were for those of adolescent age).

A surprising finding of the assessment of environmental risk factors section was that the moderate-risk group had a higher mean number of risk factors than the high-risk group. This may suggest that the number of environment risk factors decreases with an increase in severity and needs further investigation. We believe that inclusion of a population that is more likely to include all risk categories in future studies will help to clarify this association. Lastly, the sixth study, while clinical, was not conducted on actual children or adolescents but rather a chart review. The use of a large clinical population with concurrent screening of a known reliable tool may have resulted in different findings.

\section{Nursing Implications}

These studies have implications for nursing. The fifth study indicated that the two tools have potential for screening of individuals at risk for or those with actual NSSI behaviors. Although this study was not conducted using clinical participants within the focus population, it should be noted that school nurses are community-based nurses who have daily interactions with adolescents at risk for NSSI as well as those with NSSI behaviors. These participants have been essential in refinement of the two tools in preparing for testing in a clinical population.

While the number of charts reviewed was low in the sixth study, results were consistent with other studies' findings. Therefore, these tools could be useful for screening for risky behaviors such as NSSI behaviors and characteristics of poor mental health for children and adolescents. However, a final instrument development study is needed to compare the new screening tools with instruments that measure similar constructs. For instance, a study of the tools and how they perform in comparison to a known screening tool in a larger population, such as the $\mathrm{PHQ}=9$ for depression and the GAD-7 anxiety can be useful.

A power analysis demonstrated $99 \%$ sensitivity when we used a5\% alpha level and two-sided test, with a sample of 250 . Future studies will need to include larger samples, 250 or more. Such a study would also be helpful to further evaluate the significance of number of scars/wounds related to age and social determinants as well as the ability to screen risk categories. Lastly, this study was conducted in a community mental health setting where the population's risks are higher, and the tool would normally screen positive. Therefore, future studies need to focus on populations where the risk is not confounded by the setting. Considering this, future studies would be ideally located within a school setting utilizing adolescent participants.

\section{Acknowledgements}

The authors to acknowledge Dr. Katharine Bydalek, Associate Professor and Nurse Practitioner, University of South Alabama, in the initial development of both instruments.

\section{Conflict of Interest Statement}

The authors have no conflict of interest.

\section{Funding}

The school nurse study was funded in 2012 by the American Psychiatric Nurses Foundation.

\section{References}

1. Hicks KM, Hinck SM. Concept analysis of self-mutilation. Journal of Advanced Nursing. 2008; 64(4): 408-413. doi: 10.1111/j.1365-2648.2008.04822.x

2. Lee WK. Psychological characteristics of self-harming behaviors in Korean adolescents. Asian Journal of Psychiatry. 2016; 23:119-124. doi: 10.1016/j. ajp.2016.07.013

3. Whitlock J, Powers J, Eckenrode J. The virtual cutting edge: The internet and adolescent self-injury. Developmental Psychology. 2006; 42(3): 407417. doi: 10.1037/0012-1649.42.3.407.

4. Shapiro S. Addressing self-injury in the school setting. J SchNurs. 2008; 24(3):124-130. doi:10.1622/1059-8405(2008)024[0124:ASITSS]2.0.CO;2

5. Lemstra $M$, Neudorf $C, D^{\prime}$ Archy $C$, Kunst A, Warren LM, Bennett NR. et al. A systematic review of depressed mood and anxiety by SES in youth aged 10-15 years. Can J Public Health. 2008; 99(2):125-9.

6. Kelly Y, Sacker A, Del B, Francesoni M, Marmot M. What role for the home learning environment and parenting in reducing the socioeconomic gradient in child development? Findings from the Millennium Cohort Study. Arch Dis Child, 2011; 96(9):832-7. doi:10.1136/adc.2010.195917

7. Allen J, Balfour R, Bell R, Marmot M. Social determinants of mental health. Int Rev Psychiatry. 2014; 26(4):392-407. doi:10.3109/09540261.2014.928270

8. SaraffPD, Trujillo N, Pepper CM. Functions, Consequences, and Frequency of Non-suicidal Self-Injury. Psychiataric Quarterly. 2015; 86(3): 385-93. doi: 10.1007/s11126-015-9338-6

9. Baiden P, Stewart SL, Fallon B. The role of adverse childhood experiences as determinants of non-suicidal self-injury among children and adolescents referred to community and inpatient mental health settings. Child Abuse Negl. 2017; 69:163-176. doi:10.1016/j.chiabu.2017.04.011

10. Nock MK, Favazza AR. Non-suicidal self-injury: Definition and classification. In: Nock MK, ed. Understanding Non-suicidal Self-injury: Origins, Assessment, and Treatment. Washington, DC: American Psychological Association; 2009:9-18.

11. Andover M, Pepper C, Ryabchenko K, Orrico E, Gibb B et al. Self-mutilation and symptoms of depression, anxiety, and borderline personality disorder. Suicide Life Threat Behav. 2005; 35(5)581-591. doi:10.1521/ suli.2005.35.5.581

12. Lamb S. Self-harming behaviour: Raising awareness in the classroom. BJSN. 2011; 6.92-95. doi: 10.12968/bjsn.2011.6.2.92

13. Masters KJ. Temporary tattoos: A viable treatment strategy for nonsuicidal self-injury? Brown Univ Child AdolescBehav Lett. 2015; 31(8):1-6. doi:10.1002/cbl.30057

14. Stirn A, Hinz A. Tattoos, body piercings, and self-injury: Is there a connection? Investigations on a core group of participants practicing body modification. Psychotherapy Res. 2008; 18(3): 326-333. doi:10.1080/10503300701506938

15. Cleas L, Vandereycken W, Vertommen $H$. Self-care versus self-harm: Piercing, tattooing, and self-injuring in eating disorders. Eur Eat Disord Rev. 2005; 13:11-18. doi:10.1002/erv.612

16. Selby EA, Kranzler A, Fehling KB, Panza E. Nonsuicidal self-injury disorder: The path to diagnostic validity and final obstacles. ClinPsychol Rev. 2015 38:79-91. doi:10.1016/j.cpr.2015.03.003

17. Aizenman $M$, Conover-Jensen $M$. Speaking through the body: The incidences of self-injury, piercing, and tattooing among college students. J CollCouns. 2007; 12:27-43. doi:10.1002/j.2161-1882.2007.tb00004.

18. Alfonso $M$, Dedrick R. Self-injury among early adolescents. Am J Health Educ. 2010; 41(2):74-84. doi:10.1080/19325037.2010.10599131

19. Garisch J, Wilson M. Vulnerabilities to deliberate self-harm among adolescents: The role of alexithymia and victimization. Br J Clin Psychol. 2010;49(2):151-162. doi:10.1348/014466509X441709 
20. Lloyd-Richardson E, Perrine N, Dierker L, Kelley ML. Characteristics and functions of non-suicidal self-injury in a community sample of adolescents. Psychol Med. 2007; 37(8):1183-1192. doi:10.1017/S003329170700027X

21. Muehlenkamp JJ, Claes L, Harvertape L, Plener P. International prevalence of adolescent non-suicidal self-injury and deliberate self-harm. Child Adolesc Psychiatry Ment Health. 2012;30:6-10 doi:10.1186/1753-2000-6-10

22. Laye-Grindu A, Schonert-Reichl KA. Nonsuicidal self-harm among community adolescents: Understanding the "whats" and "whys" of selfharm. J Youth Adolesc. 2005; 34(5):447-458. doi:10.1007/s10964-0057262-z

23. Nock M, Prinstein M. Contextual features and behavioral functions of selfmutilation among adolescents. J Abnorm Psychol. 2005; 114(1):140-146. doi: 10.1037/0021-843X.114.1.140

24. Polanco-Roman L, Jurska J, Quiñones V, Miranda R. Brooding, reflection, and distraction: Relation to non-suicidal self-injury versus suicide attempts. Arch Suicide Res Off J IntAcad Suicide Res. 2015; 19(3):350-365. doi: 10.1080/13811118.2014.981623

25. Taliaferro LA, Muehlenkemp JJ. Factors associated with current versus lifetime self-injury among high school and college students. Suicide LifeThreat Behav. 2015; 45(1):84-97. doi:10.1111/sltb.12117

26. Favazza A. Lifetime prevalence of self-injurious behaviour among U.S. college students is 17\%. Evid Based Ment Health. 2007; 10(1):30. doi:10.1136/ebmh.10.1.30

27. Andover M, Primack J, Gibb B, Pepper C. An examination of non-suicidal self-injury in men: Do men differ from women in basic NSSI characteristics? Arch Suicide Res. 2010; 14(1):79-88. doi:10.1080/13811110903479086

28. Heath N, Ross S, Toste J, Charlebois A, Nedecheva T. Retrospective analysis of social factors and nonsuicidal self-injury among young adults. Can J Behav Sci. 2009; 41:180-186. doi:10.1037/a0015732

29. Nock M, Holmberg E, Photos V, Michel B. Self-injurious thoughts and behaviors interview: Development, validity and reliability in an adolescent sample. Psychol Assess. 2007;19(3):309-317. doi:10.1037/10403590.19.3.309

30. Ross $\mathrm{S}$, Heath N. A study of the frequency of self-mutilation in a community sample of adolescents. J Youth Adolesc. 2002; 31(3):67-77. doi:10.1023/A:1014089117419
31. Chu C, Rogers ML, Joiner TE. Cross-sectional and temporal association between non-suicidal self-injury and suicidal ideation in young adults: the explanatory roles of thwarted belongingness and perceived burdensomeness. Psychiatric Research; 2016; 246: 573-590. doi: 10.1016/j. psychres.2016.07.061

32. HaskingP. Differentiating Non-suicidal self injury and risky drinding: $A$ role for outcomes expectancies and self-efficacy beliefs. Prevention Science. 2017; 18(6): 694-703. doi: 10.1007/s11121-017-0755-7

33. Vrouva I, Fonagy $P$, Fearon $P$, Roussow $T$. The risk-taking and self-harm inventory for adolescents: Development and psychometric evaluation. Psychol Assess. 2010;22(4):852-865. doi: 10.1037/a0020583

34. Wilkinson P, Goodyer I. Non-suicidal self-injury. Eur Child Adolesc Psychiatry. 2011;20(2):103-108. doi:10.1007/s00787-010-0156-y

35. Andover $M$, Morris B, Wren A, Bruzzese $M$. The co-occurrence of nonsuicidal self-injury and attempted suicide among adolescents: Distinguishing risk factors and psychosocial correlates. Child Adolesc Psychiatry Ment Health. 2012;6: 1-7. doi:10.1186/1753-2000-6-11

36. Brackman EH, Morris BW, Andover MS. Predicting risk for suicide: A preliminary examination of non-suicidal self-injury and the acquired capability construct in a college sample. Arch Suicide Res. 2016; 20: 663676. doi./10.1080/13811118.2016.1162247

37. Whitlock J. Exner-Contens, D, \&Puringtion, A. Validity and reliability of the non-suicidal self-injury assessment test (NSSI-AT). Psychol Assess. 2014: 26(3): 935-946.

38. Williams K, Bydalek K. Adolescent self-mutilation: Diagnosis and treatment. J PsychosocNursMent Health Serv. 2007; 45(12):19-23.

39. Williams K, Bydalek K. Self-mutilation: The cutting truth. American Nurse Today. 2009; 4(8): 29-32.

40. Williams KA, Yoo W, Rikabi KK. Understanding Non-Suicidal Self-Injury Assessment in the Primary Care Setting Usefulness of the SLICE Tool. Journal of the Student National Medical Association. 2013, June 6; 21-23.

\section{Appendix \\ Appendix A: Self-Administered Risk Assessment: SARA Screening Tool @ of K. Williams, K. Bydalek, K. Hamilton \\ Instructions}

The following tool is designed to help screen for possible behaviors that may indicate the potential of current self-mutilating behaviors. This tool can be given to the adolescent students or adolescent patients in a classroom setting or clinic waiting area to be completed. Once completed it should then be reviewed by a school nurse, clinic nurse or healthcare provider. If in a classroom setting, teachers should return the tool to the school nurse for review. (Note that the gender section has been updated to reflect current gender demographic questions)

Answers of rarely, sometimes and often to questions 5-11 should be further evaluated using the SLICE tool by the school, clinic nurse, or healthcare provider.

Mark here if there is a need for evaluation using the SLICE. Yes No

Mark here if the student required assistance with reading the questions. Yes No

1. Where do you attend school? What grade are you in?

2. How old are you?

3. With which gender do you identify?

Male
__ Female
_ Transgender female
_ Transgender male
_ Gender variant/non-conforming Not listed _ Prefer not to answer (please specify)

4. How would you describe your race?

African American
Asian
White/Caucasian

5. Are you Hispanic? __ yes __ no 
6. Who do you live with? Mark all that apply:
a. Mother
c. Other Family Member
b. Father
d. Non Family Member

How often do each of these statement describe you?

5. I get angry easily or have outbursts of anger.
Never
Rarely
Sometimes
Often

6. I have nightmares/bad dreams. How true is this for you?

Never Rarely Sometimes Often

7. I eat too much or too little at times.
Never
Rarely
Sometimes
Often

8. I feel like I let myself and/or my family down in some way.
Never
Rarely
Sometimes
Often

9. I have thought about harming myself.
Never
Rarely
Sometimes
Often

This section addresses scars or wounds

10. I have scars or wounds.

No Yes

11. If you answered yes to question 10, please answer this question.

Self-inflicted wounds are those that you meant to happen.

How many of your wounds were self-inflicted?

None of them Some of them Most of them All of them

12. If you answered "Yes" to question 10, please mark on the pictures below where the wounds or scars are located.

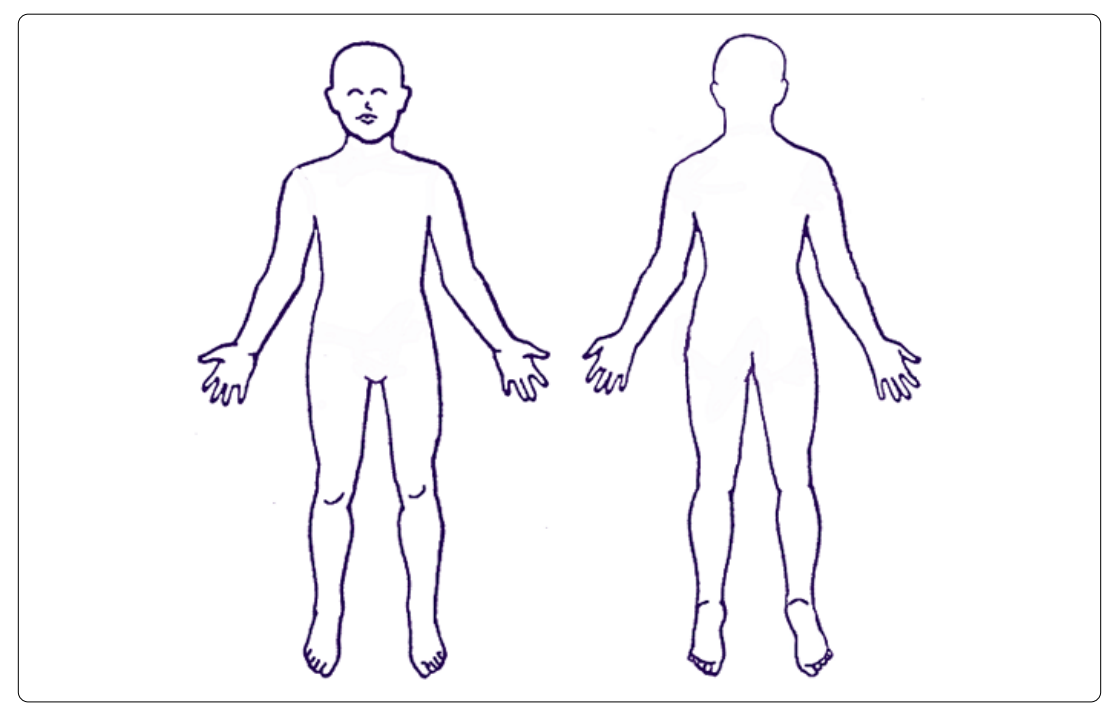

Appendix B. SLICE Tool for NSSI Risk Assessment (c) of K. Williams, K. Bydalek, K. Hamilton Information and User Instructions

This assessment tool is intended to screen for adolescents and adults in the primary care setting who may be at risk of selfmutilation or self-injury for referral to mental health services. The health care provider should obtain a history and perform a full skin assessment noting any wounds or scars. Use a non-judgmental approach when interviewing and discussing this sensitive screening content and self-harm topic.

Scars or wounds: Please briefly indicate skin findings on the assessment tool. This section can be completed after obtaining a history from the patient. One scar or cut indicates a score of 2. Multiple scares or cuts indicate a score of 4.

Links to risk factors: During the verbal history from the patient, note any risk factors/ characteristics. Add 1 point for each checked box in this section.

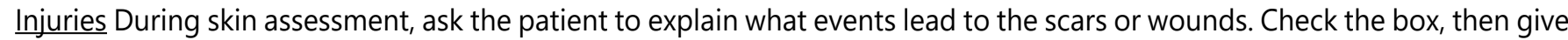
the patients description of how injury occurred. This section indicates a score of 2 
Clothing Note clothing during history or examination; be sure to indicate if clothing style is related to culture, religion, or socio-economic status. This section indicates a score of 2

Environment May obtain during the verbal history from the patient. Add a 1 point for each checked box of this section.

Total Score of $\mathrm{S}+\mathrm{L}+\mathrm{I}+\mathrm{C}+\mathrm{E}$ from page 2:

\section{Total Score Rating scale}

$0-1=$ No identified risk factors, reevaluate periodically as indicated.

2-4 = Consider giving contact information to patient of mental health services in area, revaluation in 3 months. If score is a result of patient being adolescent age and female gender, screen in 3 months only.

5-9 = needs further evaluation; contact mental health services for referral. Patient to follow-up in one week.

10 or greater $=$ Contact mental health services team to do in-facility evaluation for possible inpatient admission for treatment.

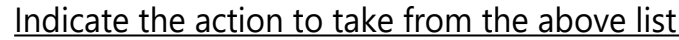

Note: Contact mental health services for immediate inpatient treatment for any indication of suicide or homicidal ideations such as written, verbal or behavioral actions.

Patient Demographics: Age___ Gender__ Race__ Ethnicity

Patient Has Past/Current NSSI Behaviors: Yes No_

DSM Axis: , II , III IV ,

Time required to complete form

Scars or wounds: (width, length \& depth/ indicate inches or centimeters) complete this section after obtaining a history from the patient. One scar are cut indicates a score of 2. Multiple scares or cuts indicate a score of 4 . Total=

Number of wounds:

012 or more

Location:

Arm Leg Abdomen Chest

Other area, specify:

Appearance:

Burns

Linear

Jagged

Smooth \& round

Irregular \& round

Links to risk factors: verbal history, note any risk factors/ characteristics. 1 point for each checked box. Total =

Adolescent (12 to 19 years)

Female

Extreme moodiness (ex. Fights at school)

Poor self-esteem

Poor impulse control

Sadness or tearfulness

Inappropriate expression of anger (ex. Aggression)

Anxiety, stress

Self disappointment

Inability to voice positive aspects in life

Feeling low or down

Under/ Over achiever

Injuries: Ask patient to explain what lead to the scars or wounds. Check box only if description of how injury does not fit. This section indicates a score of 2. Total $=$

Give dates of injuries for scars:

Patient's description of injury:

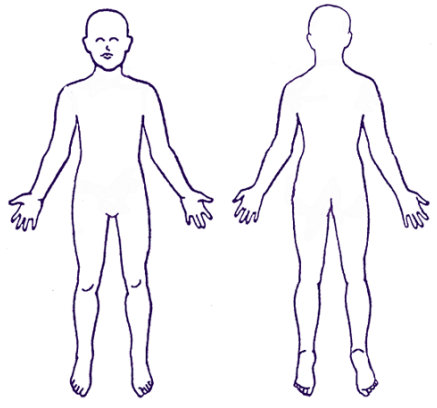

Excessive tattoos/body piercings/body gages

Eating disorder excessive/restrictive (ex. Binge eating/over eating/vomiting)

Plucking of hair on scalp, eyelashes, eyebrows or genital areas.

Use of alcohol \&/or substances, indicate

Experiencing difficulty adjusting to a new situation or issue.

Exposure to traumatic event in past or recently

Atypical development/ developmental delay

History of suicide attempt, indicate number

Currently under the care of a psychiatrist/therapist

Currently on psychiatric medications, please list below:

Clothing: Note clothing during history or examination; be sure to indicate if clothing style is related to culture, religion, or socio-economic status. This section indicates a score of 2. Total=

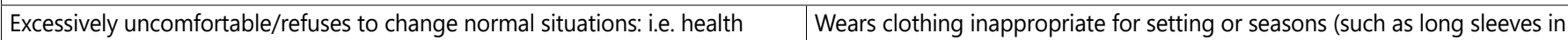
exams or sport activities in. Refusal of changing cannot be attributed to culture summer). Style cannot be attributed to culture or religion.

or religion

Environment: obtain during the verbal history from the patient. Add a 1 point for each checked box. Total=.

History/ current adolescent abuse victim

History/ current sexual abuse victim

History/ current juvenile incarceration

Parent location unknown/incarcerated

Parental/ guardian substance use/addition

Violence or turmoil in the home

Participant Code:

Feels a need for academic success

Experiencing excessive peer pressure

Living in an abusive environment (abuse directed at patient or others)

Victim of bullying/cyber bullying

Living in foster care 